\title{
Interacción Humano-Animal y Prácticas de Manejo Bovino en Subastas Colombianas
}

\author{
Human-Animal Interaction and Cattle Handling Practices in Colombian \\ Livestock Auctions
}

\author{
Lucia Herrán', Marlyn Romero ${ }^{2.4}$, Lorena Herrán ${ }^{3}$
}

\section{Resumen}

\begin{abstract}
El objetivo del estudio fue evaluar las interacciones humano-animal (IHA) y las prácticas de manejo en subastas de Medellín, Colombia. Se recopilaron datos de cuatro subastas ganaderas realizadas en junio de 2014. Se evaluaron 720 bovinos, los cuales, durante el pesaje y la conducción se subdividieron en 241 lotes y 28 grupos de lotes, respectivamente. Se caracterizaron los hallazgos en inspección veterinaria, así como las interacciones táctiles, auditivas y visuales de los manejadores y las respuestas conductuales en los bovinos. En la inspección veterinaria, en el 19.5\% de los lotes se hicieron observaciones, con predominio de heridas abiertas (38.3\%). En el área de pesaje se evidenciaron 824 interacciones, predominando las visuales $(44.1 \%)$ sobre las táctiles (30.1\%) y auditivas (25.8\%); sin embargo, el 73.0, 66.4 y 88.4\% de los animales no presentaron respuesta conductual a las interacciones visuales, táctiles y auditivas, respectivamente. En la conducción a corrales se registraron 214 interacciones, con predominio de las táctiles (42.5\%) sobre las auditivas (30.8\%) y visuales (26.6\%); sin embargo, la mayoría de lotes solo presentó respuesta conductual a las interacciones táctiles (68\%). Las interacciones positivas de manejo como el uso de silbidos, ondear el instrumento y alzar las manos generaron pocas respuestas conductuales y manejos más tranquilos de los animales. Por otro lado, golpear, punzar y gritar provocó mayor reactividad y comportamientos agonísticos en los animales.
\end{abstract}

Palabras clave: bienestar animal; interacciones; respuestas conductuales; manejadores

\footnotetext{
${ }^{1}$ Facultad de Medicina, Universidad CES, Medellín, Colombia

${ }^{2}$ Grupo de Investigación en Ciencias Veterinarias CIENVET, Departamento de Salud Animal, Programa de Medicina Veterinaria y Zootecnia, Facultad de Ciencias Agropecuarias, Universidad de Caldas, Manizales, Colombia

${ }^{3}$ Facultad de Ciencias Administrativas y Agropecuarias, Corporación Universitaria Lasallista, Caldas, Colombia

${ }^{4}$ E-mail: marlyn.romero@ucaldas.edu.co
}

Recibido: 11 de noviembre de 2016

Aceptado para publicación: 26 de mayo de 2017 
The objective of the study was to evaluate the human-animal interaction (HAI) and the handling practices at a livestock market in Medellin, Colombia. Data was collected at four livestock auctions in June 2014. A total of 720 bovines were distributed into 241 and 28 groups during the weighing and driving to the corral respectively. Findings during the veterinary inspection, as well as tactile, auditory and visual interactions of cattle handlers and behavioral responses of cattle were evaluated. In the veterinary inspection, $19.5 \%$ of the groups were observed, especially due to open wounds (38.3\%). In the weighing area, there were 824 interactions, predominantly visual (44.1\%) and then tactile $(30.1 \%)$ and auditory (25.8\%); however, $73.0,66.4$ and $88.4 \%$ of the animals had no behavioral response to visual, tactile and auditory interactions, respectively. Twenty-four interactions were recorded during driving to corrals, with tactile predominance $(42.5 \%)$ over auditory $(30.8 \%)$ and visual $(26.6 \%)$; however, most of the groups presented only behavioral response to tactile interactions $(68 \%)$. Positive handling interactions such as whistling, waving the instrument, and raising hands generated few behavioral responses and quieter animal handling. On the other hand, hitting, punching, and screaming provoked greater reactivity and agonistic behaviors in the animals.

Key words: animal welfare; interactions; behavioral responses; handlers

\section{INTRODUCCIÓN}

Dentro de la cadena logística bovina existe un eslabón conformado por las ferias comerciales y las subastas ganaderas, en donde se desarrollan eventos que congregan a productores y compradores para establecer alianzas estratégicas a corto plazo, identificar oportunidades y comparar la calidad de sus animales con otros del mercado; así como para estudiar el comportamiento de la oferta y los precios de los bovinos en pie (DANE, 2013; Miranda-de la Lama, 2014). Estos mercados desempeñan un rol importante en la industria ganadera y en las comunidades rurales, y ofrecen oportunidades laborales a un gran número de personas (Robinson y Chistley, 2007). Sin embargo, se han descrito como desventajas, la dificultad de mantener un programa de trazabilidad de los animales que allí se comercializan, el insuficiente control sanitario de las autoridades competentes, el incremento del riesgo de la transmisión de enfermedades y un potencial impacto negativo en el bienestar animal y en la calidad e inocuidad de la carne de estos animales cuando llegan a las plantas de sacrificio (Brigas-Poulin et al., 2006; Gregory, 2008).

Con relación al bienestar animal, cuando los bovinos se comercializan a través de las ferias y subastas comerciales, se presentan interacciones humano-animal (IHA) principalmente negativas, debido a una mayor reactividad de los bovinos expuestos a ambientes novedosos (Waiblinger et al., 2006), lo que genera factores estresores que desencadenan pérdidas de peso, animales fatigados, con ayuno prolongado y deshidratación; aumentando la probabilidad de contusiones y lesiones, cuando se comparan con los bovinos que ingresan a las plantas de sacrificio, procedentes directamente de la granja (Strappini et al., 2012; Romero et al., 2013). De igual manera, las respuestas agonísticas de los bovinos dificultan el trabajo del personal, favorecen el retraso de las labores, pudiendo ocasionar disminución de la satisfacción laboral, desmotivación, falta de compromiso y problemas de autoestima (Breuer et 
al., 2003; Waiblinger et al., 2006) y de manera ocasional, riesgos ocupacionales como lesiones e incluso la muerte por accidentes (Le Neindre et al., 1996).

Las subastas ganaderas en Colombia son consideradas como los lugares de concentración de todo tipo de especies animales comerciales, cuya finalidad es la venta para el levante y engorde posterior en predios o para el sacrificio para consumo; o con la finalidad de exhibición y promoción de especies y razas de animales para reproducción y mejoramiento genético (ICA, 2001). El conocimiento de las conductas que caracterizan la IHA puede ser una herramienta importante para identificar riesgos operacionales, orientar la adopción de prácticas de manejo más eficientes y brindar lineamientos para fortalecer los programas de entrenamiento y capacitación del personal (Strappini et al., 2007; Romero et al., 2011. Así mismo, se presentan como una forma práctica, económica y no invasiva para evaluar el bienestar animal en estos mercados (Waiblinger et al., 2006).

El objetivo del presente estudio fue evaluar la IHA en una subasta ganadera, durante el pesaje y la conducción, mediante las interacciones utilizadas por los manejadores y la respuesta de los bovinos a estas interacciones.

\section{Materiales y Métodos}

Se realizó un estudio transversal en una subasta ganadera física durante el mes de junio de 2014. Fueron incluidos todos los lotes bovinos que ingresaron a las cuatro subastas del mes entre 07:00 y 11:00 horas, excluyéndose lotes bovinos sin movilidad (fatiga, calambres, fracturas, otros). El estudio contó con el aval del Comité Operativo de Investigaciones de la Facultad de Medicina de la Universidad CES (Acta N. ${ }^{\circ} 98$, Código de Proyecto N. ${ }^{\circ} 852$ de 20 de agosto de 2013), considerado como una actividad de investigación con mínimo riesgo.

\section{Localización Geográfica}

La subasta ganadera está localizada en Medellín, departamento de Antioquia $\left(6^{\circ} 132\right.$

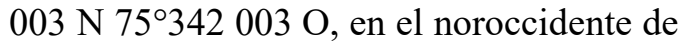
Colombia), zona que se caracteriza por un clima de bosque lluvioso tropical, con un promedio anual de lluvias de $2060 \mathrm{~mm}$, temperatura promedio anual de $16.6^{\circ} \mathrm{C}$ y una altitud de $2550 \mathrm{msnm}$. La subasta contaba con cinco rampas de descarga en material de hormigón y pisos antideslizantes, conectados por un flujo continuo con los corrales de recepción, pesaje $(3 \times 5 \mathrm{~m})$ y una serie de pasillos (76.3 $\mathrm{m}$ de largo por $2.7 \mathrm{~m}$ de ancho), que conectaban con 32 corrales de alojamiento. Los corrales contaban con suministro de agua ad libitum, desprovistos de techos y otras condiciones de sombra. El suministro de alimento se hacía a discreción de los propietarios de los animales.

\section{Personal}

El personal vinculado a las labores de manejo animal en la subasta evaluada era joven, menor de 38 años. La mitad de ellos con nivel de escolaridad básica primaria y más de 10 años de experiencia ejerciendo esta labor. Sus motivaciones principales para ser manejadores de animales fueron la responsabilidad familiar y el deseo de superación personal.

\section{Manejo Animal}

En las instalaciones de la feria ganadera se desarrollaron las actividades de recepción, pesaje, inspección veterinaria y comercialización en subasta de ganado los días martes, para un total de cuatro subastas mensuales y un ingreso promedio de 2000 bovinos de diversas categorías productivas.

Los bovinos evaluados fueron conducidos por los manejadores mediante el uso de bandera o escobilla (cuyo extremo era utilizado para golpear a los animales) y dispuestos en corrales de selección donde se subdividían en lotes más pequeños ( $\mathrm{n}=241$ lotes) 
de acuerdo a las necesidades comerciales de los propietarios. A continuación, eran conducidos a un embudo donde se marcaban, pesaban y se efectuaba la inspección sanitaria por un médico veterinario para determinar la condición física del lote. Posteriormente, los bovinos eran alojados en un corral de espera donde se mezclaban y conformaban lotes con un mayor número de bovinos $(\mathrm{n}=28)$ y finalmente conducidos por dos manejadores a los corrales de espera previo al proceso de subasta. En los lotes se presentaba mezcla de sexo y categoría productiva.

\section{Interacción Humano-Animal y Prácticas de Manejo}

Se evaluaron 720 bovinos que correspondieron a las categorías productivas de hembras de vientre, hembras de levante, vacas horras, toros, vaca parida con su cría, machos de ceba y machos de levante; así como ocho manejadores con capacitaciones previas en bienestar animal realizadas por la empresa. Se excluyeron los bovinos no ambulatorios por fatiga o lesión osteomuscular. Durante el pesaje y la conducción los bovinos se subdividieron en 241 y 28 lotes, respectivamente. Los datos fueron recolectados mediante el uso de instrumentos simplificados de observación con mediciones basadas en el animal (IHA y respuestas conductuales) (Breuer et al., 2003; Waiblingler et al., 2006) y en los recursos (manejo) estructurados y validados con las condiciones locales del mercado (Minka y Аyo, 2007).

La evaluación de la interacción IHA se realizó por cuatro observadores entrenados, sin interferir con la rutina normal de trabajo, durante el pesaje y la conducción de los bovinos, usando la metodología descrita por Breuer et al. (2003) y Waiblinger et al. (2006), pero adaptado a la especie bovina y a las características comerciales de la subasta; debido a esto, se disminuyeron los tiempos de observación y no se establecieron tiem- pos de adaptación. Durante el pesaje, se ubicaron dos observadores, donde cada uno evaluó el desempeño de un manejador, desde el momento en que se abrió la puerta del embudo de inspección para conducir el lote al corral de pesaje hasta que el último animal del lote salió del corral de pesaje e ingresó al corral de espera. Así mismo, durante la conducción se ubicaron dos observadores, donde cada uno realizó la evaluación a un manejador, desde el momento en que conducía, separaba o movía el grupo de varios lotes bovinos, hasta que estos ingresaban a los corrales de alojamiento (Figura 1).

Se observaron las prácticas de manejo del personal (Cuadro 1), la respuesta conductual de los bovinos, tales como caídas (si alguna parte del animal, además de las pezuñas, toca el suelo), resbaladas (pérdida del equilibrio temporal por desplazamiento de una o varias extremidades), retroceso (el animal da más de tres pasos hacia atrás) y vocalizaciones intencionales (no incluye los jadeos, suspiros o gemidos) (Grandin, 2010).

Así mismo, se incluyeron las posturas agresivas de los animales frente a los manejadores (Cuadro 2) cuando se observaron los patrones de varias conductas en una sola interacción, de acuerdo con los lineamientos dados por Horwitz et al. (2002), pero adaptados a la especie bovina y a las características comerciales de la subasta; debido a esto, se disminuyeron los tiempos de observación y no se establecieron tiempos de adaptación. En el momento en que el manejador conducía, separaba o movía un lote durante el pesaje o un grupo de varios lotes durante la conducción a los corrales, se observaba durante 30 segundos la interacción que el manejador empleaba y por 10 segundos la respuesta conductual bovina (Breuer et al., 2003; de Vries, 2011; Cobo, 2013).

El observador no inició una nueva medición sin haber culminado el tiempo de observación anterior. 


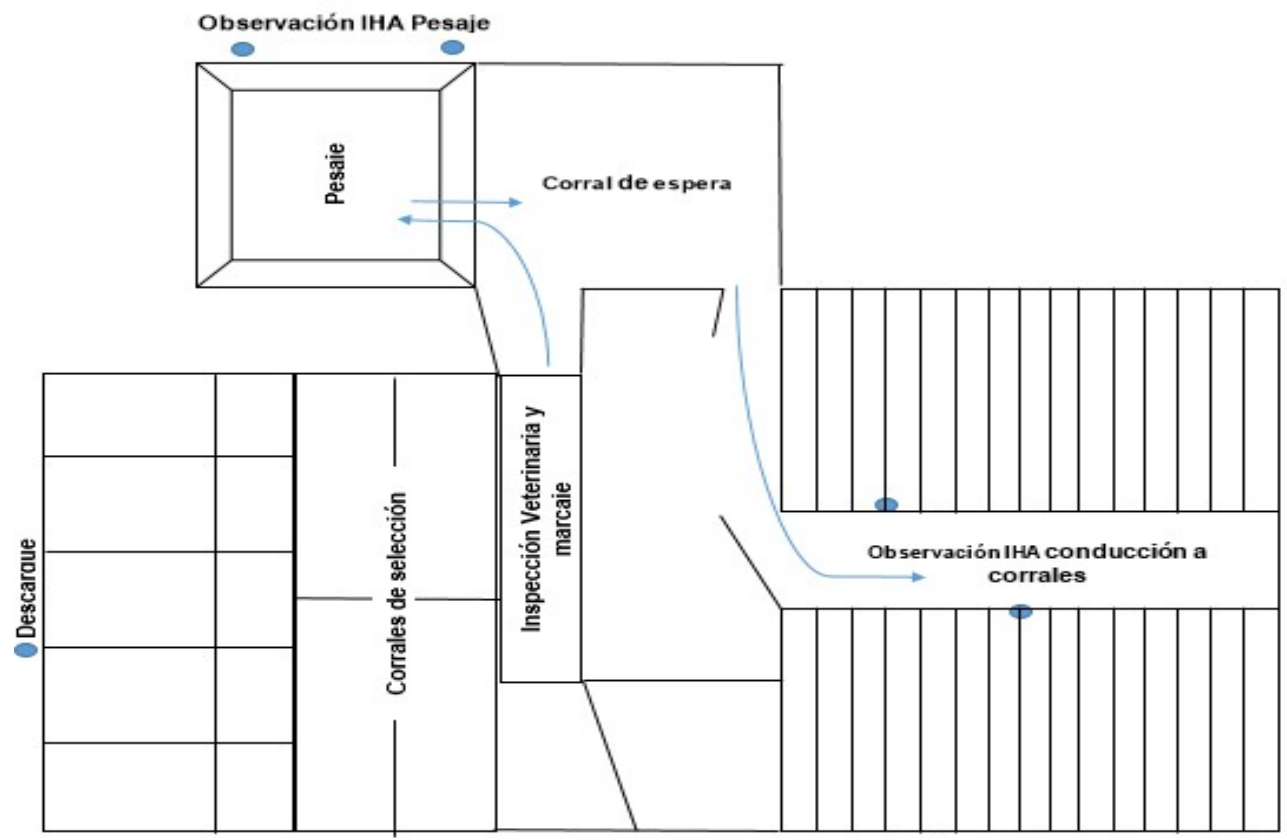

Figura 1. Esquema de la localización de los observadores en la evaluación de las interacciones humano-animal (IHA) durante el pesaje y la conducción en la subasta ganadera en Medellín, Colombia

Cuadro 1. Indicadores de las interacciones táctiles, auditivas y visuales usadas por los manejadores de ganado en una subasta ganadera en Medellín, Colombia

\begin{tabular}{|c|c|c|}
\hline Interacción & Acción & Descripción \\
\hline \multirow[t]{3}{*}{ Táctiles } & Golpear & $\begin{array}{l}\text { Impactar al bovino con una parte del cuerpo del } \\
\text { manejador o con el instrumento de manejo }\end{array}$ \\
\hline & Punzar & $\begin{array}{l}\text { Utilizar instrumentos punzantes de forma localizada en } \\
\text { el animal }\end{array}$ \\
\hline & Torsión de cola & $\begin{array}{l}\text { Torcer la cola del animal, tomándola con las manos y } \\
\text { doblándola en un ángulo agudo }\end{array}$ \\
\hline \multirow[t]{3}{*}{ Auditivas } & Gritar & Emisión de un sonido fuerte por parte del manejador \\
\hline & Silbar & Sonido agudo emitido por la boca del manejador \\
\hline & Sonidos artificiales & $\begin{array}{l}\text { Ruidos fuertes producidos por instrumentos como palos } \\
\text { y metales }\end{array}$ \\
\hline \multirow[t]{3}{*}{ Visuales } & Ondear & $\begin{array}{l}\text { Mover suavemente de un lado para el otro el } \\
\text { instrumento de manejo (palo con extremo en forma de } \\
\text { pompón o banderas) }\end{array}$ \\
\hline & Bloqueo & $\begin{array}{l}\text { Realizar bloqueo de visual del animal con algún } \\
\text { material físico o con el cuerpo del manejador para } \\
\text { impedir que avance }\end{array}$ \\
\hline & Alzar manos & $\begin{array}{l}\text { Levantar las manos detrás del animal con el objetivo de } \\
\text { aparentar un mayor tamaño }\end{array}$ \\
\hline
\end{tabular}

Fuente: Breuer et al. (2003) y Waiblinger et al. (2006) 


\section{Análisis Estadístico}

Se realizó un análisis univariado para describir las IHA táctiles, auditivas y visuales de los manejadores y la respuesta conductual de los bovinos por lote (pesaje) y grupo de lotes (conducción). El análisis bivariado se hizo por medio de las correlaciones de Pearson para estudiar la consistencia entre el número de interacciones de los manejadores y el número de respuestas conductuales. Se realizó un análisis de componentes principales con rotación varimáx para encontrar el resumen de variables comunes de la IHA durante el pesaje y la conducción. La consistencia interna entre las variables incluidas en el análisis de componentes principales se realizó por medio del coeficiente de fiabilidad Alfa de Cronbach. Los datos fueron procesados con el programa estadístico SPSS (Statistical Package for the Social Sciences) v. 21.0 (Licencia Universidad CES).

\section{Resultados}

\section{Caracterización de los Lotes}

Los lotes se clasificaron durante la inspección veterinaria y se estableció que el $31.5 \%(\mathrm{n}=75)$ estaba conformado por hembras de vientre, $23.2 \%(n=56)$ por hembras de levante, $19.5 \%(\mathrm{n}=47)$ por machos de levante, $9.5 \%(\mathrm{n}=23)$ por machos de ceba, $8.3 \%$ $(n=20)$ por hembras y sus crías, $4.1 \%(n=10)$ por vacas horras, $2.5 \%(n=6)$ por lotes de diferentes categorías productivas y $1.7 \%$ $(n=4)$ por toros.

El $80.5 \%(n=194)$ de lotes bovinos no presentó hallazgos de significancia clínica a la inspección veterinaria y en el $19.5 \%(n=47)$ se encontraron heridas abiertas $(n=18$; $38.3 \%)$, descorne reciente $(n=8 ; 17.0 \%)$, infecciones $(\mathrm{n}=6 ; 12.8 \%)$, restricción del amamantamiento de la cría $(n=6 ; 12.8 \%)$, hernias $(\mathrm{n}=5 ; 10.6 \%)$, hembras próximas al parto $(\mathrm{n}=2$; $4.3 \%)$ y hembras caquécticas $(\mathrm{n}=2 ; 4.3 \%)$.

\section{Interacción Humano-Animal y Prácticas de Manejo}

Los 720 bovinos estuvieron distribuidos en 241 lotes, con un promedio de $3 \pm 3.5$ (rango 1-22) por lote, donde se registraron 824 interacciones, predominando las visuales (44.1\%), seguidas de las táctiles (30.1\%) y las auditivas (25.8\%) (Cuadro 3).

La mayor proporción de bovinos no presentaron respuestas conductuales a las interacciones usadas por los manejadores durante el pesaje y las respuestas observadas presentaron coeficientes de correlación de Pearson débiles $(r<0.27)$. La agresividad ambivalente fue la respuesta más frecuente (Cuadro 4).

En la conducción a los corrales de alojamiento se observaron 28 grupos $(n=720$ bovinos), con un promedio por grupo de 25.2 \pm 16.3 (rango 3-67). Se registraron 214 interacciones, de las cuales predominaron las táctiles (42.5\%), seguidas de las auditivas $(30.8 \%)$ y las visuales (26.6\%) (Cuadro 5).

La mayoría de lotes no presentó respuesta conductual durante el manejo en la conducción a las interacciones visuales y auditivas, pero se observaron reacciones a las interacciones táctiles $(\mathrm{n}=19 ; 68 \%)$. Retrocesos, caídas y resbalones fueron las respuestas más frecuentes (Cuadro 6). Se observaron correlaciones significativas entre el total de las interacciones táctiles $(\mathrm{r}=0.6$; $\mathrm{p}<0.01)$ y las visuales $(\mathrm{r}=0.42 ; \mathrm{p}<0.05)$ y las respuestas conductuales de los bovinos, más no así con las interacciones auditivas $(\mathrm{r}=0.35$; $\mathrm{p}>0.05$ ).

El análisis de componentes principales identificó dos componentes que definieron la IHA de los lotes durante el pesaje. Estos componentes explicaron el 57.1\% de la varianza del constructo, resultado adecuado y estadísticamente significativo $(\mathrm{KMO}=0.57$; Prueba de Bartlett's X2 $=230.24$; valor $\mathrm{P}=0.000$ ). La confiabilidad del constructo fue 
Cuadro 2. Descripción de categorías emergentes en la respuesta conductual agresiva de los bovinos en una subasta ganadera en Medellín, Colombia

\begin{tabular}{ll}
\hline \multicolumn{1}{c}{ Postura } & \multicolumn{1}{c}{ Descripción } \\
\hline $\begin{array}{l}\text { Agresividad con postura } \\
\text { ofensiva }\end{array}$ & $\begin{array}{l}\text { Patrones agresivos claramente definidos dirigidos hacia el } \\
\text { manejador (frente al manejador y mirada fija, intentar embestir, } \\
\text { vocalizaciones, resistencia, patadas). }\end{array}$ \\
$\begin{array}{l}\text { Agresividad con postura } \\
\text { defensiva }\end{array}$ & $\begin{array}{l}\text { No intenta acercarse al manejador, procura evitar el contacto } \\
\text { directo y no son patrones de agresión directos o dirigidos } \\
\text { (retroceder, saltos, coleos, correr). }\end{array}$ \\
$\begin{array}{l}\text { Agresividad con postura } \\
\text { ambivalente }\end{array}$ & $\begin{array}{l}\text { Cuando se observan ambas posturas de agresividad (ofensiva y } \\
\text { defensiva en una misma respuesta ante la IHA). }\end{array}$ \\
\hline
\end{tabular}

Fuente: Horwitz et al. (2002)

Cuadro 3. Análisis descriptivo de las interacciones usadas por los manejadores de bovinos durante el pesaje en una subasta ganadera en Medellín, Colombia

\begin{tabular}{clcccc}
\hline Variable & \multicolumn{1}{c}{ Categoría } & $\mathrm{N}$ & Promedio & D.E & Rango \\
\hline IHA táctiles & Golpear & 216 & 1.17 & 0.43 & $1-4$ \\
& Torsión de cola & 10 & 2.50 & 0.58 & $2-3$ \\
& Punzar & 22 & 1.83 & 0.39 & $1-2$ \\
\cline { 2 - 6 } IHA auditivas & Total & 248 & 1.03 & 0.65 & $1-4$ \\
\cline { 2 - 6 } & Gritar & 17 & 1.00 & 0 & $1-1$ \\
& Silbar & 190 & 1.16 & 0.37 & $1-2$ \\
& Sonidos artificiales & 6 & 3.00 & 0 & $3-3$ \\
\cline { 2 - 6 } IHA visuales & Total & 213 & 0.88 & 0.61 & $1-3$ \\
\cline { 2 - 6 } & Ondear & 316 & 1.81 & 0.43 & $1-3$ \\
& Bloqueo & 4 & 1.00 & 0 & $1-1$ \\
& Alzar manos & 43 & 1.07 & 0.27 & $1-2$ \\
\cline { 2 - 6 } & Total & 363 & 1.51 & 0.68 & $1-3$ \\
\hline
\end{tabular}

determinada mediante el cálculo del alfa de Cronbach 0.61. El primer componente lo conformaron las interacciones táctiles, auditivas y visuales de los manejadores, definidas para este estudio como «conducta de los manejadores» y el segundo componente conformado por la respuesta conductual de los bovinos, definida como «conducta de los bovinos», todas con correlaciones fuertes y directas (Cuadro 7) (Figura 2).

Durante la conducción se identificaron dos componentes que definieron la IHA. Estos componentes explicaron el $63.6 \%$ de la 
Cuadro 4. Distribución porcentual del comportamiento de bovinos como respuesta a las interacciones de los manejadores durante el pesaje ( $\mathrm{n}=241$ lotes, 720 bovinos) en una subasta ganadera en Medellín, Colombia

\begin{tabular}{lccc}
\hline \multirow{2}{*}{ Comportamiento bovino } & \multicolumn{3}{c}{ Interacciones de los manejadores } \\
\cline { 2 - 4 } & $\begin{array}{c}\text { Táctiles } \\
\mathrm{n}(\%)\end{array}$ & $\begin{array}{c}\text { Visuales } \\
\mathrm{n}(\%)\end{array}$ & $\begin{array}{c}\text { Auditivas } \\
\mathrm{n}(\%)\end{array}$ \\
\hline Sin respuesta conductual & $160(66.4)$ & $176(73.0)$ & $213(88.4)$ \\
Vocalizaciones & $3(1.2)$ & $4(1.7)$ & $1(0.4)$ \\
Caídas & $4(1.7)$ & $9(3.7)$ & - \\
Resbalones & $10(4.1)$ & $12(5.0)$ & $4(1.7)$ \\
Retrocesos & $10(4.1)$ & $2(0.8)$ & - \\
Agresividad ofensiva & $7(2.9)$ & $8(3.3)$ & $7(2.9)$ \\
Agresividad defensiva & $17(7.1)$ & $5(2.1)$ & $4(1.7)$ \\
Agresividad ambivalente & $30(12.4)$ & $25(10.4)$ & $12(5.0)$ \\
\hline
\end{tabular}

Cuadro 5. Descripción del número de interacciones usadas por los manejadores durante la conducción a corrales en una subasta ganadera en Medellín, Colombia

\begin{tabular}{clcccc}
\hline Variable & \multicolumn{1}{c}{ Categoría } & $\mathrm{n}$ & Promedio & D.E. & Rango \\
\hline \multirow{5}{*}{ IHA táctiles } & Golpear & 72 & 3.43 & 1.63 & $1-7$ \\
& Torsión de cola & 13 & 4.33 & 1.15 & $3-5$ \\
& Punzar & 6 & 1.50 & 0.58 & $1-2$ \\
\cline { 2 - 6 } IHA auditivas & Total & 91 & 3.25 & 1.64 & $1-7$ \\
\cline { 2 - 6 } & Gritar & 44 & 3.14 & 0.95 & $2-5$ \\
& Silbar & 8 & 1.60 & 0.55 & $1-2$ \\
& Sonidos artificiales & 14 & 3.50 & 2.38 & $1-6$ \\
\cline { 2 - 6 } IHA visuales & Total & 66 & 2.36 & 1.66 & $1-6$ \\
\cline { 2 - 6 } & Ondear & 3 & & & \\
& Bloqueo & 32 & 3.20 & 1.48 & $1-6$ \\
& Alzar manos & 22 & 1.57 & 0.76 & $1-3$ \\
\cline { 2 - 6 } & Total & 57 & 2.04 & 1.45 & $1-6$ \\
\hline
\end{tabular}


Cuadro 6. Distribución porcentual del comportamiento bovino como respuesta a las interacciones de los manejadores durante la conducción ( $\mathrm{n}=28$ lotes) en una subasta ganadera en Medellín, Colombia

\begin{tabular}{lccc}
\hline Categorías & $\begin{array}{c}\text { Respuesta } \\
\text { IHA táctil } \\
\mathrm{n}(\%)\end{array}$ & $\begin{array}{c}\text { Respuesta } \\
\text { IHA visual } \\
\mathrm{n}(\%)\end{array}$ & $\begin{array}{c}\text { Respuesta } \\
\text { IHA auditiva } \\
\mathrm{n}(\%)\end{array}$ \\
\hline Sin respuesta conductual & $9(32.1)$ & $17(60.7)$ & $17(60.7)$ \\
Caídas & $3(10.7)$ & $3(10.7)$ & $2(7.1)$ \\
Resbalones & $2(7.1)$ & $3(10.7)$ & $3(10.7)$ \\
Retrocesos & $5(17.9)$ & $1(3.6)$ & $3(10.7)$ \\
Agresividad ofensiva & $3(10.7)$ & $1(3.6)$ & $2(7.1)$ \\
Agresividad defensiva & $4(14.3)$ & $2(7.1)$ & $1(3.6)$ \\
Agresividad ambivalente & $2(7.1)$ & $1(3.6)$ & - \\
\hline
\end{tabular}

Cuadro 7. Resultados del análisis de componentes principales que definieron la calidad de las interacciones humano-animal (IHA) durante el pesaje y la conducción en una subasta ganadera en Medellín, Colombia

\begin{tabular}{lcccc}
\hline & \multicolumn{2}{c}{ Pesaje } & \multicolumn{2}{c}{ Conducción } \\
\cline { 2 - 5 } Variables & $\begin{array}{c}\text { Componente 1 } \\
\text { Conducta } \\
\text { manejadores }\end{array}$ & $\begin{array}{c}\text { Componente } 2 \\
\text { Conducta } \\
\text { bovina }\end{array}$ & $\begin{array}{c}\text { Componente 1 } \\
\text { Calidad } \\
\text { positiva }\end{array}$ & $\begin{array}{c}\text { Componente 2 } \\
\text { Calidad } \\
\text { negativa }\end{array}$ \\
\hline $\begin{array}{l}\text { Respuesta } \\
\text { conductual a } \\
\text { interacción táctil }\end{array}$ & 0.304 & 0.702 & -0.041 & 0.857 \\
$\begin{array}{l}\text { Interacción táctil } \\
\begin{array}{l}\text { Interacción } \\
\text { auditiva }\end{array}\end{array}$ & 0.755 & 0.144 & 0.161 & 0.872 \\
$\begin{array}{l}\text { Respuesta } \\
\text { conductual a } \\
\text { interacción }\end{array}$ & 0.872 & 0.001 & 0.856 & 0.033 \\
$\begin{array}{l}\text { auditiva } \\
\begin{array}{l}\text { Interacción visual } \\
\text { Respuesta } \\
\text { conductual a } \\
\text { interacción visual }\end{array}\end{array}$ & 0.198 & 0.773 & 0.328 & 0.559 \\
\hline
\end{tabular}


variabilidad del constructo, resultado adecuado y estadísticamente significativo $(\mathrm{KMO}=0.60$; Prueba de Bartlett's X2 $=37.40$; valor $\mathrm{P}=0.001)$. La confiabilidad del constructo fue determinada mediante el cálculo del alfa de Cronbach 0.70 (Figura 2).

\section{Discusión}

En los lotes predominaron las categorías de hembras de vientre, así como las hembras y machos de levante, los cuales tienden a ser más reactivos al manejo (Lensink et al., 2001). De otra parte, los lotes de toros, tuvieron poca presencia en el estudio, pero un gran impacto por las dificultades que representa manejarlos, dada su dominancia en grupos sociales, especialmente cuando hay presencia de hembras (Grandin, 2007).

A pesar de que la mayor proporción de los bovinos evaluados se encontró en buenas condiciones de salud durante la inspección veterinaria y cumplió con este requerimiento sanitario (ICA, 2001; DANE, 2013), predominaron las heridas abiertas en los lotes que registraron hallazgos en la inspección, resultados concordantes con los descritos por de
Vries (2011) al evaluar ocho subastas ganaderas en el sur de Chile. La presencia de heridas abiertas es un indicador de bienestar animal poco descrito en subastas de animales (Alam et al., 2010; de Vries, 2011; IPCVA, 2012); no obstante, es relevante porque afecta las IHA por el dolor y suponen experiencias negativas previas (Weeks et al., 2002).

Se detectaron bovinos con lesiones infectadas, en estado de caquexia y hembras próximas al parto, aspectos que son prohibidos por la legislación sanitaria colombiana vigente (ICA, 2007), pero que son condiciones habituales durante el transporte comercial de bovinos (ICA, 2001; Romero et al., 2011). La presencia de crías con restricción del amamantamiento es un hallazgo que es necesario evaluar en próximas investigaciones, porque impide la ingesta de leche y de agua, produce deshidratación, estrés, hambre y angustia de los terneros, aspecto que aumenta la respuesta emocional y afecta de manera negativa su bienestar (Strappini et al., 2012).

Los lotes de animales durante el pesaje fueron pequeños y, en su mayoría, provenían del mismo grupo social; aspecto que podría explicar las pocas respuestas conductuales a
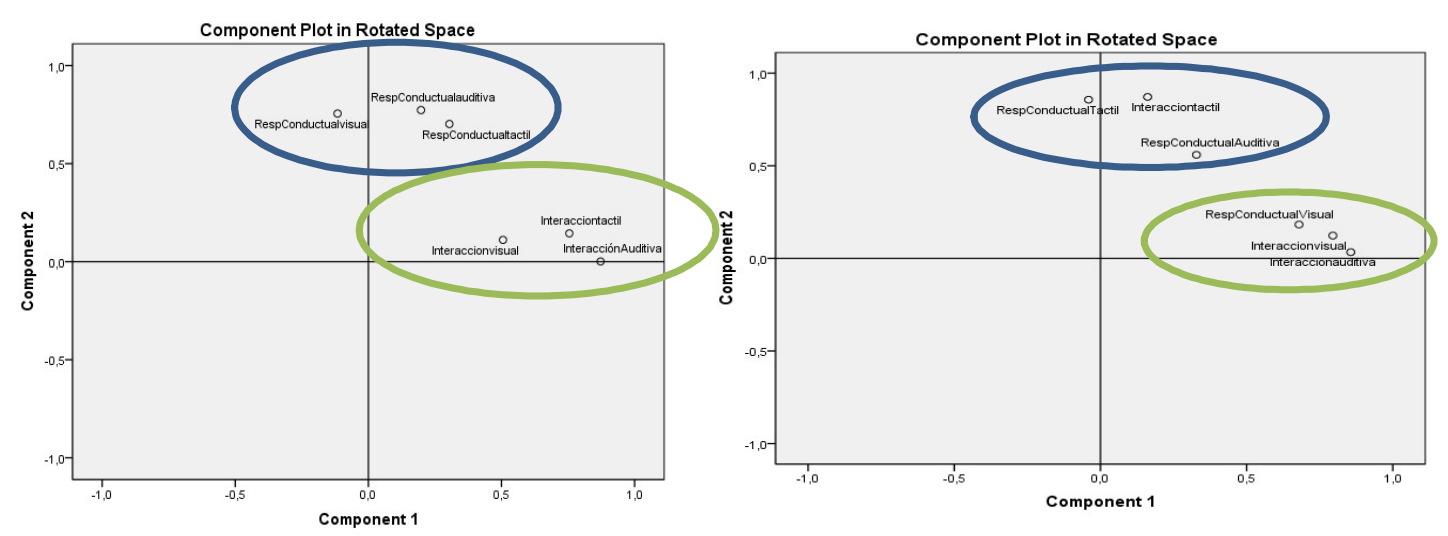

Figura 2. Análisis de los componentes principales de las variables que intervinieron en las interacciones humano-animal (IHA) durante el pesaje (izquierda) y la conducción (derecha) de ganado bovino en una subasta ganadera en Medellín, Colombia 
las interacciones auditivas y visuales, que facilitaron el manejo de los lotes en esta área (de Vries, 2011). Sin embargo, las respuestas conductuales agonísticas fueron mayores que aquellas reportadas en plantas de beneficio colombianas (Cobo, 2013), pero se encuentran acordes con autores que evaluaron la IHA en subastas chilenas (de Vries, 2011).

Las vocalizaciones presentaron una frecuencia inferior del $3 \%$, aspecto que cumple con los estándares internacionales recomendados para auditorías de bienestar animal en plantas americanas (Grandin, 2010). Por otro lado, las caídas y resbalones, como respuesta a interacciones táctiles y auditivas, estuvieron por encima del 1 y $3 \%$, respectivamente, lo cual podría ser una respuesta al estrés generado por el manejo (Grandin, 2010) o por la presencia de pisos resbalosos, húmedos, sin recubrimientos antideslizantes (de Vries, 2011).

Las interacciones táctiles frecuentes durante el pesaje pueden estar relacionadas con una actitud de alerta para prevenir accidentes por parte del manejador, dadas las limitaciones de espacio en esta área. Se ha descrito que el manejador es quien determina el número y la naturaleza de las interacciones (Breuer et al., 2003; Waiblinger et al., 2006) y que los comportamientos agonísticos de los animales desencadenan reacciones de miedo de los manejadores e incrementan el uso de interacciones táctiles (de Vries, 2011). Los animales que recibieron estas interacciones mostraron posturas agresivas y las conductas de retroceder, resbalar, caer y vocalizar; descritas también por otros autores en Colombia (Romero et al., 2012; Cobo, 2013). En auditorías de bienestar animal no son aceptadas las interacciones táctiles como punzar y la torsión de la cola (Grandin, 2010), observadas en este estudio.

Las interacciones auditivas estuvieron presentes, en especial los silbidos; sin embargo, la mayoría de los lotes de animales continuaron con su marcha calmada, sin signos de miedo (Breuer et al., 2003; Grandin,
2010; IPCVA, 2012), aspecto que evidencia la naturaleza positiva de la interacción (de Vries, 2011; Hemsworth y Coleman, 2011; Cobo, 2013). En Colombia, el uso de silbido es una práctica cultural arraigada en los manejadores de ganado (Cobo, 2013); así mismo, las buenas prácticas pecuarias fomentan su uso en lugar de interacciones táctiles (ICA, 2007), por lo cual, la habituación de los bovinos a esta experiencia previa podría ser la explicación de estos resultados (Breuer et al., 2003; Waiblinger et al., 2006).

El uso de gritos y la emisión de sonidos artificiales fueron usados con baja frecuencia para la conducción del ganado en el presente estudio. Estos resultados difieren de los encontrados en un estudio reciente realizado en trabajadores de una planta de beneficio de Colombia, donde su uso fue una interacción tan negativa como el uso del tábano eléctrico en zonas sensibles del animal, la cual generó respuestas agonísticas y aumentó las concentraciones de cortisol sanguíneo (Cobo, 2013).

La baja asociación estadística entre las interacciones visuales de los manejadores y las respuestas conductuales de los bovinos, podría deberse al estrecho contacto entre los manejadores y los bovinos en las instalaciones de la subasta, que favorecía la invasión de la zona de fuga (Waiblinger et al., 2006). Si bien la literatura no describe la medida de zona de fuga aceptada como adecuada, investigaciones en el Reino Unido y en Chile reportan valores entre 0.7 y $3.2 \mathrm{~m}$ (Pajor et al., 2000; Arraño et al., 2007), las cuales teniendo en cuenta el tamaño del área de pesaje de la subasta y el promedio de animales por lote, probablemente fueron invadidas. No se evidenció el uso de las palmas, patadas y del tábano eléctrico, interacciones descritas en otras investigaciones (Grandin, 1998; Breuer et al., 2003; Cobo, 2013; Hemsworth y Coleman, 2011).

Las débiles correlaciones entre las respuestas conductuales de los bovinos frente a las interacciones de los manejadores durante el pesaje pudieron atribuirse a la influencia 
de otras variables del entorno que no fueron evaluadas como la ansiedad inducida por la novedad, el hambre, los manejos previos y construcciones anexas a la subasta (Waiblinger et al., 2006). Son escasos los estudios que prueben específicamente la validez discriminante de las medidas de IHA y, por lo tanto, se requieren investigaciones que aborden este aspecto. En este estudio, estas variables no se controlaron por las características comerciales reales de la subasta.

De acuerdo con el análisis de componentes principales, se sugiere que durante el pesaje estuvieron presentes situaciones confusas, donde predominaron la novedad, la posible invasión de la zona de fuga y el manejo de lotes muy pequeños que afectaron la condición gregaria de los bovinos e impidieron un adecuado reconocimiento mutuo con los manejadores, requisito para que una IHA se desarrolle de manera efectiva (Waiblinger et al., 2006). Por tanto, la IHA pudo basarse principalmente por los patrones de conductas que tanto el manejador como el animal expresaron de manera individual; teniendo en cuenta que, en una IHA, la percepción entre el manejador y los animales se expresa en su mutuo comportamiento, donde también se pueden generalizar experiencias previas que actúan como retroalimentación de la interacción (Hemsworth y Coleman, 2011). Para mitigar el impacto potencial de este tipo de variables que confunden el comportamiento de los bovinos, se recomienda un periodo de habituación (Waiblinger et al., 2006).

Este estudio se realizó bajo las condiciones comerciales reales de la subasta, por tanto, no fue posible implementar un periodo previo de habituación. La conducción de los bovinos hacia los corrales por medio de grupos conformados por diferentes lotes, sexos y categorías productivas, generó un ambiente novedoso y poco familiar para los animales (Lensink et al., 2001; Waiblinger et al., 2006; Hemsworth y Coleman, 2011). Se han realizado estudios para calcular las distancias promedio de las áreas de fuga que han demostrado su importancia en el manejo bovino
(Arraño et al., 2007; Martínez et al., 2010). El tamaño y la conformación de grupos sociales diferentes pudo desencadenar el uso de interacciones táctiles por parte de los manejadores durante todas las conducciones observadas, así como la mayor presencia de respuestas agresivas y reversadas por parte de los bovinos (de Vries, 2011; IPCVA, 2012). Golpear al animal y los tratos abusivos como punzar y la torsión de cola, presentaron resultados concordantes con los reportados en Colombia (Cobo, 2013), Chile (de Vries, 2011) y Bangladesh (Alam et al., 2010). Las interacciones táctiles por parte de los manejadores, siempre constituyen experiencias negativas para los animales (Singer y Casal, 1999).

Durante la conducción, las vocalizaciones no se presentaron en ninguna de las interacciones, a diferencia del área de pesaje y de otras investigaciones (IPCVA, 2012), pero las caídas y resbalones estuvieron por encima de los indicadores de bienestar permitidos (Grandin, 2010).

La interacción auditiva gritar se considera de naturaleza negativa (Lensink et al., 2001) y fue la más frecuente en el área de conducción, la cual estuvo asociada con respuestas de los bovinos como resbalar, retroceder y la manifestación de agresividad con postura defensiva. Es conocido que el oído de los bovinos es más sensible que el de los humanos, por lo que los gritos les produce miedo (Grandin, 2007) y mayor excitación, en especial, cuando no pueden ubicar el origen del sonido (Grandin, 1998). Así mismo, el ambiente de la subasta con la presencia de música, perifoneo y gritos constantes de personas, puede ser fuente de estrés para los animales y un factor de confusión en el análisis de los resultados durante la conducción (Waiblinger et al., 2006).

Las interacciones visuales más frecuentes fueron los bloqueos y alzar manos, manejos acordes y aceptados para una conducción adecuada de grupos de bovinos (Grandin, 1998; de Vries, 2011). No obstante, la con- 
ducción es un evento novedoso para los bovinos, que genera desafíos, emociones y aptitudes de alerta (Grandin, 2007), de allí que se presentaron respuestas conductuales ante estas interacciones como caídas, resbalones y agresividad con postura defensiva.

El análisis de componentes principales durante la conducción, explicó la IHA mediante la calidad positiva o negativa de la interacción. El primer componente «Calidad positiva» reflejó las interacciones y respuestas conductuales positivas que favorecieron el bienestar animal de los grupos de lotes durante la conducción. De esta manera, las interacciones auditivas y visuales por parte de los manejadores permitieron que las respuestas conductuales de los bovinos no reflejaran miedo o estrés y así pudieron ser conducidos de manera calmada. El segundo componente «Calidad negativa» reflejó las interacciones y respuestas conductuales negativas que generaron deterioro en el bienestar de los bovinos durante la conducción. De esta manera, las interacciones táctiles por parte de los manejadores manifestaron respuestas conductuales agonísticas y, para el caso de las respuestas conductuales a interacciones auditivas, el factor ambiental pudo generar un efecto aditivo, que reforzó la calidad negativa de la interacción (Pond et al., 2006; Waiblinger et al., 2006). Estos resultados concuerdan con Hemsworth y Coleman (2011), quienes reportan que es el manejador el que generalmente define la calidad e inicia la interacción, pudiendo inconscientemente emitir señales resultantes de miedo o tranquilidad para el animal; por tanto, diferencias sutiles en el comportamiento humano pueden ser cruciales.

\section{Conclusiones}

- Las interacciones positivas de manejo como el uso de silbidos, ondear el instrumento y alzar las manos generaron pocas respuestas conductuales y manejos más tranquilos de los animales.
- Golpear, punzar y gritar provocó mayor reactividad y comportamientos agonísticos de los bovinos.

\section{Agradecimientos}

Los autores agradecen a la Central Ganadera S.A. en gerencia del Dr. Jorge Mario Calle, por su valiosa disposición de los recursos físicos y operativos para la realización del estudio; al Dr. Juan Tobón por su apoyo profesional y sus aportes a la ejecución del proyecto; e indudablemente a los manejadores «arrieros» quienes tuvieron la disposición de atender todos los requerimientos y permitieron que esta investigación se llevara a cabo.

\section{Literatura Citada}

1. Alam NG, Gregory MA, Jabbar MS, Uddin ASMG, Kibria A, Silva-Fletcher A. 2010. Skin injuries identified in cattle and water buffaloes at livestock markets in Bangladesh. Vet Rec 167: 415-419. doi: 10.1136/vr.c3301

2. Arraño C, Báez A, Flor E, Whay HR, Tadich N. 2007. Estudio preliminar del uso de un protocolo para evaluar el bienestar de vacas lecheras usando observaciones basadas en el animal. Arch Med Vet 39: 239-245. doi: 10.4067/S0301732X2007000300007

3. Breuer K, Hemsworth P, Coleman GJ. 2003. The effect of positive or negative handling on the behavioural and physiological responses of nonlactating heifers. Appl Anim Behav Sci 84: 3-22. doi: 10.1016/S0168-1591(03)00146-1

4. Brigas-Poulin M, Thompson RA, Chriel M, Mortensen S, Greiner M. 2006. Network analysis of Danish cattle industry trade patterns as an evaluation of risk potential for disease spread. Prev Vet Med 76: 11-39. doi: 10.1016/ j.prevetmed.2006.04.004

5. Cobo CG. 2013. Evaluación de la interacción hombre-animal como indicador de bienestar animal durante el 
presacrificio bovino. Tesis de Maestría. Manizales: Universidad de Caldas. $71 \mathrm{p}$.

6. DANE, Ministerio de Agricultura y Desarrollo Rural. 2013. Caracterización de las ferias ganaderas en el país. Boletín mensual Insumos y Factores Asociados a la Producción Agropecuaria.

7. de Vries M. 2011. Human-animal relationship at Chilean livestock markets. MSc Tesis. The Netherlands: Wageningen University. 53 p.

8. Grandin T. 1998. Review: reducing handling stress improves both productivity and welfare. Prof Anim Scientist 14: 1-10. doi: 10.15232/S10807446(15)31783-6

9. Grandin T. 2007. Livestock handling and transport. $3^{\text {rd }}$ ed. UK: CABI. 386 p.

10. Grandin T. 2010. Auditing animal welfare at slaughter plants. Meat Sci 86: 56-65. doi: 10.1016/j.meatsci.2010. 04.022

11. Gregory NG. 2008. Animal welfare at markets and during transport and slaughter. Meat Sci 80: 2-11. doi: 10.1016/ j.meatsci.2008.05.019

12. Hemsworth PH, Coleman GC. 2011. Human-livestock interactions: the stockperson and the productivity and welfare of intensively farmed animals. $2^{\text {nd }}$ ed. Wallingford, UK: CAB International. $194 \mathrm{p}$.

13. Horwitz DF, Mills DS, Heath S. 2002. BSAVA Manual of canine and feline behavioural medicine. Quedgeley, Gloucester: BSAVA. 288 p.

14. [ICA] Instituto Colombiano Agropecuario. 2001. Resolución No. 02495 del 10 de septiembre de 2001, por la cual se establecen los requisitos sanitarios para la realización de concentraciones de animales en el Territorio $\mathrm{Na}$ cional. [Internet]. Disponible en: http:// www.ica.gov.co/getattachment/ 71a4662d-1979-4cfc-ac88-82574cde713a/2495.aspx

15. [ICA] Instituto Colombiano Agropecuario. 2007. Reglamento sobre las condiciones sanitarias y de inocuidad del ganado bovino y bufalino en la producción primaria. Diario Oficial de la República. Resolución $N^{\circ} 002341$.

16. [ICA] Instituto Colombiano Agropecuario. 2007. Resolución N. ${ }^{\circ} 002341$ del 23 de agosto de 2007, por la cual se reglamentan las condiciones sanitarias y de inocuidad en la producción primaria de ganado bovino y bufalino destinado al sacrificio para consumo humano. [Internet]. Disponible en: http:// www.ica.gov.co/getattachment/ 0 b 5 d e 556 - c b 4 a - 43 a 8 - a 27 a cd9a2064b1ab/2341.aspx

17. [IPCVA] Instituto de Promoción de la Carne Vacuna Argentina. 2012. Evaluación del trato de los animales en instalaciones de remate ferias. Cuadernillo Técnico No. 12. Universidad Nacional del Litoral. [Internet]. Disponible en: http://www.ipcva.com.ar/files/ct12.pdf

18. Le Neindre $P$, Boivin $X$, Boissy $A$. 1996. Handling of extensively kept animals. Appl Anim Behav Sci 49: 7381. doi: 10.1016/0168-1591(95)00669-9

19. Lensink BJ, Fernandez $X$, Gozzi $G$, Florand L, Veissier I. 2002. The inûuence of farmers' behavior on calves' reactions to transport and quality of veal meat. J Anim Sci 79: 642-652.

20. Martínez $E$, Carrasco $G A A$, Galindo MF, Muñoz PME, López de Buen L. 2010. Evaluación del bienestar animal e indicadores conductuales de salud y productivos en bovinos de lechería tropical. En: XLVI Reunión Nacional de Investigación Pecuaria, Campeche, México.

21. Minka NS, Ayo JO. 2007. Effects of loading behaviour and road transport stress on traumatic injuries in cattle transported by road during the hot-dry season. Livest Sci 107: 91-95. doi: 10.1016/j.livsci.2006.10.013

22. Miranda-de la Lama GC, Villarroel M, María GA. 2014. Livestock transport from the perspective of the preslaughter logistic chain: a review. Meat Sci 98: 9-20. doi: 10.1016/j.meatsci. 2014.04.005 
23. Pajor EA, Rushen J, De Pasillé AMB. 2000. Aversion learning techniques to evaluate dairy cattle handling practices. Appl Anim Behav Sci 69: 89-102. doi: 10.1016/S0168-1591(00)00119-2

24. Pond WG, Pond KR, Ducar P. 2006. Introducción a la ciencia animal. Zaragoza: Ed Acribia. 664 p.

25. Robinson SE, Christley RM. 2007. Exploring the role of auction markets in cattle movements within Great Britain. Prev Vet Med 81: 21-37. doi: 10.1016/ j.prevetmed.2007.04.011

26. Romero M, Sánchez J, Gutierrez C. 2011. Evaluación de prácticas de bienestar animal durante el transporte de bovinos para sacrificio. Rev Salud Pública 13: 684-690.

27. Romero MH, Cobo CG, González LM. 2013. Estudio de indicadores conductuales para evaluar el descargue de bovinos en plantas de beneficio. Luna Azul 37: 10-17.

28. Romero MH, Uribe LF, Sánchez JA. 2012. Evaluación de la conducta y las prácticas de manejo durante el sacrificio bovino, como indicadores de bienestar animal. CES Med Vet Zootec 7: 22-29.
29. Singer P, Casal P. 1999. Liberación animal. Madrid: Ed Trotta. 334 p.

30. Strappini A, Gallo C, Cáraves $M$, Barrientos A, Allende R, Chacón F, Briones I. 2006. Relevamiento preliminar del transporte de ganado bovino y ovino en Chile: vehículos y manejo de los animales durante la descarga. En: Res XXXII Congreso Anual de la Sociedad Chilena de Producción Animal. Frutillar. Chile.

31. Strappini AC, Metz JHM, Gallo C, Frankena $K$, Vargas $R$, de Freslon I, Kemp B. 2012. Bruises in culled cows: where and how are they inflicted? Animal 7: 485-491. doi: 10.1017/ S1751731112001863

32. Waiblinger S, Boivin X, Pedesen V, Tosi MV, Janczak AM, Visser EK, Jones RB. 2006. Assessing the human-animal relationship in farmed species: a critical review. Appl Anim Behav Sci 101: 185242. doi: 10.1016/j.applanim.2006.02.001

33. Weeks CA, McNally PW, Warris PD. 2002. Influence of the design of facilities at auction markets and animal handling procedures on bruising in cattle. Vet Rec 150: 743-748. doi: 10.1136/ vr.150.24.743 